\title{
Efficient Hybrid (ANTC) Approach for Edge Detection Technique
}

\author{
Navdeep Kaur \\ M.Tech Scholar, Department of Electronics and \\ Communication, Amritsar College of Engineering \& \\ Technology, Amritsar
}

\author{
Vijay Kumar \\ Prof.ECE (Principal), \\ Amritsar College of Engineering \& Technology, \\ Amritsar
}

\begin{abstract}
These are many techniques are used in earlier research work many techniques are used to solved the problem of edge detection but all of them having disadvantages in form of speed, noise etc. So the hybrid technique used to producing better result than the other edge detection techniques like canny, prewit, sobel and Roberts. The hybrid approach based edge detection can successfully reduce the poor speed issue with ant colony optimization. The color based gradients has the ability to remove the effect of the false edges while preserving the potential edges.
\end{abstract}

\section{Keywords}

Image Segmentation, Edge Detection, Various Edge Detection Approaches, Hybrid Approach.

\section{INTRODUCTION}

This image segmentation can function as element of a visible in to components or agencies, which fit various things or facets of objects. Every pixel in a picture is given to a minimum of among a couple of these categories. An excellent segmentation is usually one wherever:

- Pixels in the identical celebration have attached grayscale of multi-variety charges and sort a linked place,

- Neighboring pixels that can be found in a number of agencies have dissimilar values.

The solutions to segmentation, termed thresholding, edgebased techniques and region-based strategies.

\subsection{Thresholding}

In thresholding, pixels with charges less than 128 have formerly been put in to one celebration, and the remainders have formerly been put in to an added different category. The restricts between bordering pixels in a number of agencies has been superimposed in brilliant on the first image. It could be observed that the thresholding has correctly segmented the photograph in to both key fiber types

\subsection{Edge based Segmentation}

In edge-based segmentation, accomplishment filtration is placed on the photograph; pixels are identified as place or non-edge regarding the filtration productivity, and pixels that aren't separated by excellent details are given to the identical category.

\subsection{Region based Segmentation}

Fundamentally, region-based segmentation calculations perform iteratively by quantity together pixels which are neighbors and have attached charges and breaking up bits of pixels which are dissimilar in value.

\section{EDGE DETECTION}

Edge recognition of color pictures is actually among the crucial tasks in digital image processing. Presently, the manners of color images edge detection are generally same as the manner of grayscale images [1]. The more and more special list have begun originally to examine the medial edge recognition of shade picture. Some specialists supplies the medial edge detection of color image, individuals have created plenty of practices [2-13], and including another three forms mainly. The original one is that along with image is transformed proper right into a grey-scale picture, then portion popularity is performed by the gradient individual or huge change operator. Then the other one is that side recognition of color picture is performed by the gradient individual or positive change individual to every way (red, green and blue) of shade picture respectively, the outcomes of every way are mixed to the stops of shade picture. Fundamentally, along with ends are transformed proper right into a grey-scale picture. And another is on the foundation of the quaternion and vector, like the methods about color huge positive change and vector dot solution, etc. [10,11].

Even though the formerly mentioned three variety practices are certain to get the medial part of shade picture, they nevertheless involve some shortcomings. The original variety technique might eliminate unfinished portion data of color image when transforming along with image into grey-scale image. The next variety technique might include more synthetic criteria in the unpredictable components, because it might be a stage whose gray or dull gets the most convert in the gradient means for a particular way (i.e. portion stage of a particular channel) by the gradient individual or positive change individual; nonetheless, the reason why blended three purposes might probably possibly maybe not be an advantage stage of the entire picture. And another one is on the foundation of the integer-order differential operator. Usually, these practices frequently find prevents on the foundation of the non-continuity of pixel's energy positively and the mathematical options that come with pixels aren't regarded, while they emphasize on the practicality and the intensive usefulness [14].

Typically, particular demands by Canny [9] applied since the usefulness evaluation normal of side recognition algorithm that is really a great localization and decreased multiplicity. Canny's results are really a good normal when it's applied to get the reliability of edge recognition algorithm. For a specific side recognition individual, the 3 facets of particular demands are regular limitation and hard to think about, thus each edge recognition algorithm has their particular collection and focus. In this paper, we discussed a new method for color pictures using color gradients and mix method focused canny edge detector as well as ant colony optimization. 


\subsection{Various Edge Detection Approaches}

There are various edge detection methods. Some of them are discussed in this paper.

\subsubsection{Canny Edge Detector}

Canny algorithms just one more technique for edge recognition, that has the benefits of excellent SNR (Signal-toNoise Ratio), correct position and portion reduction [3]. None the less, the first canny algorithm is done on gray scale images. The evaluation on shaded picture is essential and useful, therefore, this record presents an improved color picture results by using canny algorithm. The normal canny algorithm on gray scale pictures might be split directly into four pieces: picture smoothing, gradient computation, nonmaxima withdrawal, side willpower and connection [11].

\subsubsection{Ant Colony Optimization}

ACO as a swarm intelligence technique has been used to fix edges. This Approach (ACO) produces the perceptual understanding of images for eliminating edge features. ACObased edge recognition strategies may change the photograph level rates to the pheromone rates outstanding by produced bugs in the images. Relating with one of these put pheromone rates, the photograph edges may be acknowledged [14].

\subsubsection{Combination of Color Components for Edge Detection}

That is done through an option of the very most correct areas from every shade space. First, study the actions of the conventional canny part alarm located with this color spaces. For this type of function, use of canny part alarm on each section then identify essentially the most correct types which is regarded as most readily useful persons for a potential mixed space. The duty is really a quantitative general study that utilizes newest effective mathematical methods for part quality assessment. Here correctly need to select essentially the most relevant color areas using quantitative evaluation patterns predicated on similarly, made picture (with simple line edge) and complicated real-world color photographs with unique identified surface.

\subsubsection{Area Optimizes FPGA}

Some researchers has target with this particular study for color image edge recognition applying Sobel client for movie detective applications (frame price $25 \mathrm{fps}$ to $50 \mathrm{fps}$ ) to be able to total in real-time and at the same time frame body consume the little area. In implementation, three pieces of Sobel client are projected for shaded picture edge recognition. This could need the FPGA methods (area) three times in comparison to gray-scale picture area recognition. The platform utilizes easy Sobel client part for examine edge for most three color channels. That's attained by functioning the Sobel client part at the same time frame price of three times the pixel knowledge begin quantity and multiplexing the inputs. This platform is interfaced with camera application purpose and present operator purpose and utilized on FPGA ML 510 (Virtex - 5 FX130T) platform. It robustly registers along with edge in real-time at price of 50fps [8].

\subsubsection{Hybrid Edge Detector}

Many area alarm, function as gradient-based practices or zerocrossing methods, needs convolving an aesthetic with a kernel to compute gradients or zero-crossing. On the foundation of the link involving the convolution, a selection is then made individuals to whether a pixel is an edge or not. The duty of this strategy mainly includes three methods: gradient magnitude computation, thresholding and the last one is second order derivative price computation. The gradientbased practices provide very little grasp on picture sound and portion location. The second-order derivative strategy tends to exaggerate sound doubly much. Some type of sound reduction is involve [9].

\section{RESULTS AND DISCUSSION}

This shows the outputs of the existing approach i.e. (ACO) as well as the proposed approach i.e. Hybrid (ACO \& Canny) Approach. The following results completely shows that the proposed hybrid approach gives better outputs because edges are detected in an beneficial manner.

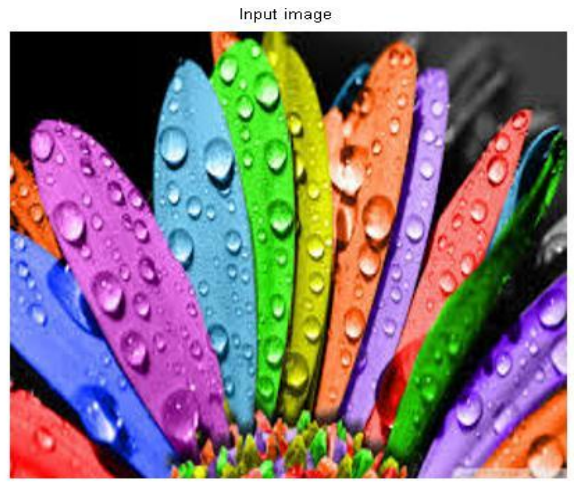

(a) Input Image

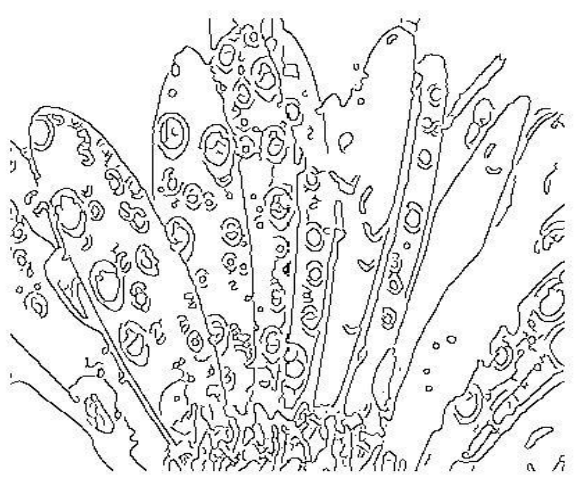

(b) ACO Approach

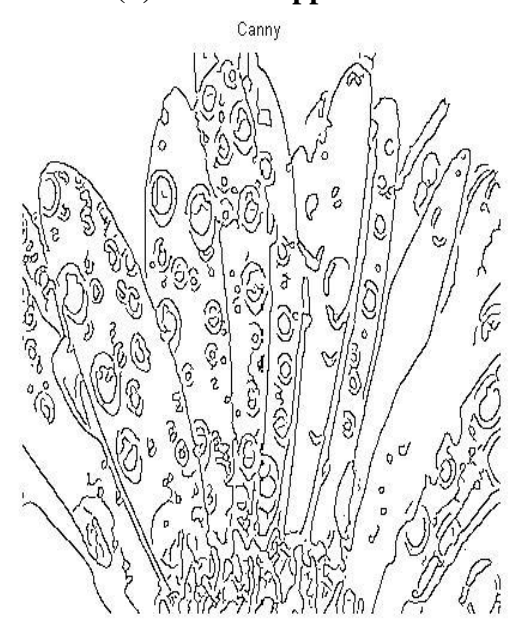

(c) Canny Approach 


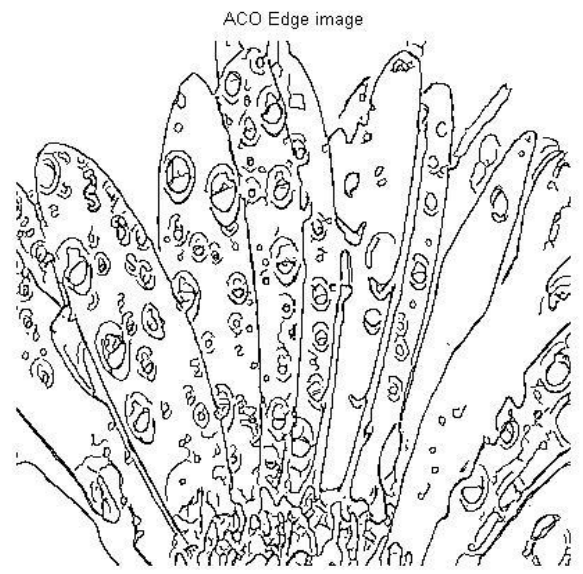

(d) ANTC Approach

Fig 1: Edge detection results of picture 10

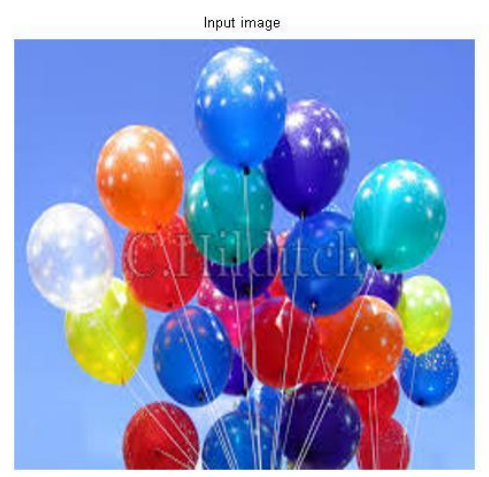

(a) Input Image

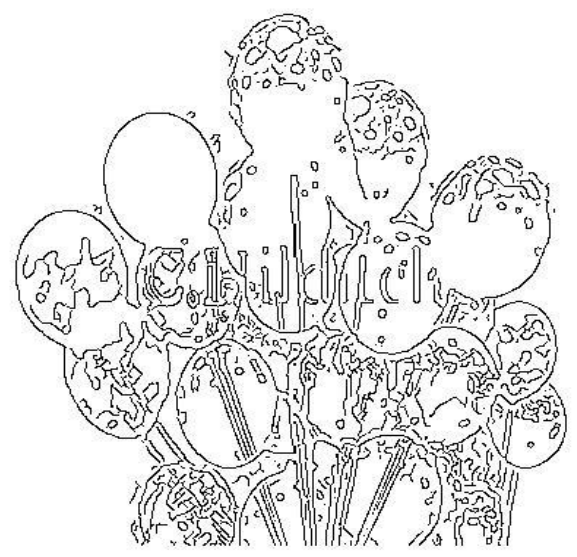

(b) ACO Approach

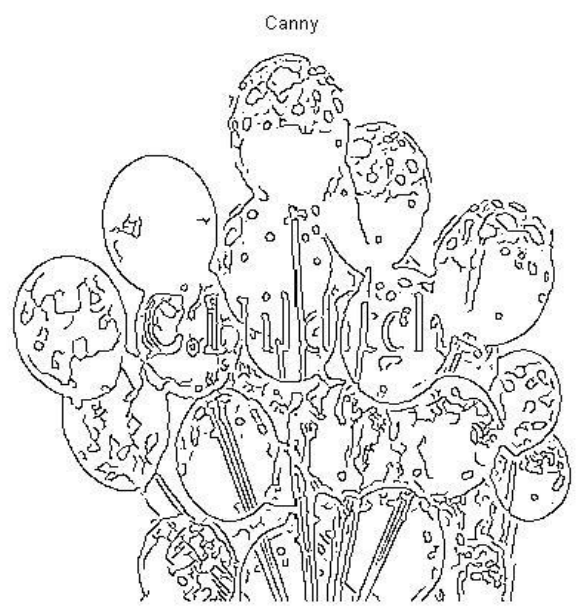

(c) Canny Approach

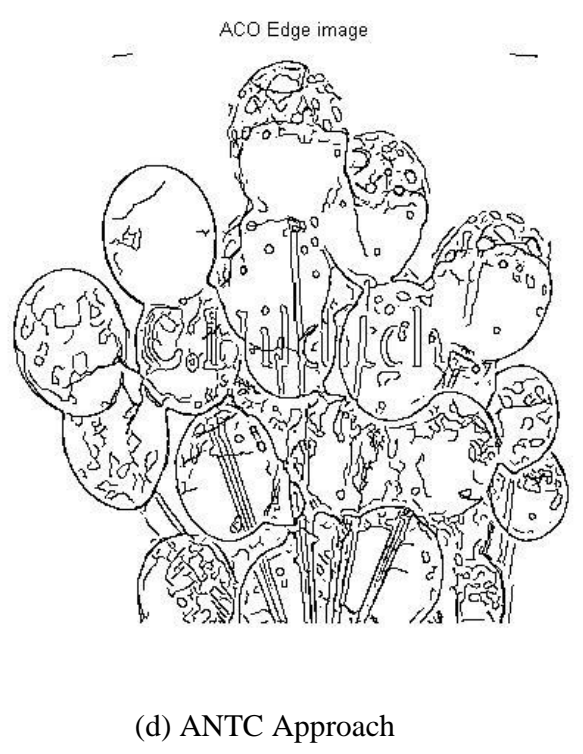

Fig 2: Edge detection results of image 9

\subsection{Performance Evaluation}

This section shows the results of our proposed technique ANTC as well as existing techniques.

\subsubsection{Mean Square Error}

Mean square error is to compute an error signal by subtracting the test signal from the reference, and then computing the average energy of the error signal. Their units are shown in mean error per pixels.

TABLE 1

\begin{tabular}{|c|c|c|c|}
\hline Input & MEAN SQUARE ERROR \\
Images & Canny & ACO & $\begin{array}{c}\text { Hybrid } \\
\text { (ANTC) }\end{array}$ \\
\hline Image1 & 0.2389 & 0.1379 & 0.0173 \\
\hline Image2 & 0.1501 & 0.2669 & 0.0182 \\
\hline
\end{tabular}




\begin{tabular}{|c|l|l|l|}
\hline Image3 & 0.2562 & 0.1428 & 0.0176 \\
\hline Image4 & 0.2205 & 0.1194 & 0.0205 \\
\hline Image5 & 0.2437 & 0.1268 & 0.0050 \\
\hline Image6 & 0.0378 & 0.0664 & 0.0073 \\
\hline Image7 & 0.2972 & 0.0650 & 0.0097 \\
\hline Image8 & 0.2567 & 0.1027 & 0.0093 \\
\hline Image9 & 0.1393 & 0.1219 & 0.0169 \\
\hline Image10 & 0.1498 & 0.1664 & 0.0198 \\
\hline
\end{tabular}

Table 3: shows the line graph of the existing as well as the proposed approach. It shows that the values of proposed approach are lower than the existing approach.

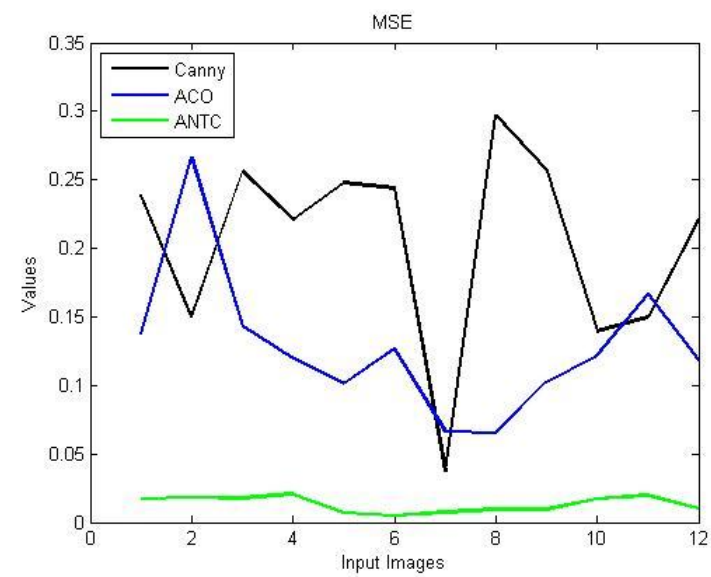

Fig 3: line graph of MSE

\subsubsection{Pratt's Figure of Merit}

The Pratt evaluate requires a "gold standard" - that offers perfect or ideal edges for the images. After using a standard edge recognition algorithm for this purpose best comparison results of edge detection are shown by this parameter. The distance between an actual and an ideal edge is just the distance in the picture, computed using Pythagoras' theorem on their coordinates. This assumption based on matching up corresponding edges, which may not be trivial. There is a single summation because the sums across edges are done, not across pixels.

TABLE 2

PRATT'S FIGURE OF MERIT

\begin{tabular}{|c|l|l|l|}
\hline Input & Canny & ACO & $\begin{array}{c}\text { Hybrid } \\
\text { (ANTC) }\end{array}$ \\
\hline Images & 0.6921 & 0.6470 & 0.8038 \\
\hline Image2 & 0.7114 & 0.6795 & 0.8017 \\
\hline Image3 & 0.6913 & 0.6950 & 0.8017 \\
\hline Image4 & 0.6999 & 0.6868 & 0.7978 \\
\hline
\end{tabular}

\begin{tabular}{|c|l|l|l|}
\hline Image5 & 0.7122 & 0.6312 & 0.8140 \\
\hline Image6 & 0.6372 & 0.7925 & 0.8117 \\
\hline Image7 & 0.6391 & 0.6948 & 0.7961 \\
\hline Image8 & 0.6680 & 0.7223 & 0.8077 \\
\hline Image9 & 0.7244 & 0.7106 & 0.8029 \\
\hline Image10 & 0.7351 & 0.7384 & 0.8038 \\
\hline
\end{tabular}

Table 4: shows the line graph of the existing as well as the proposed approach. It shows that the values of proposed approach are higher than the existing approach.

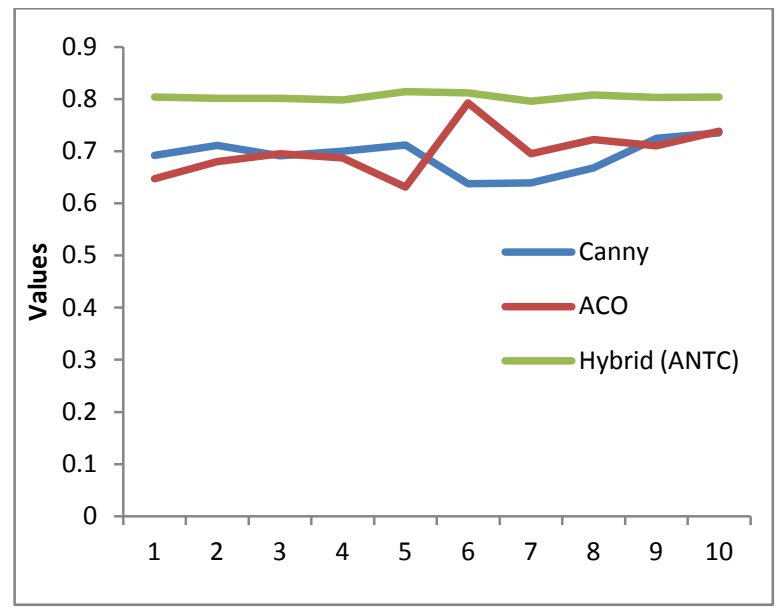

Fig 4: line graph of Pratt's figure of merit

\section{CONCLUSION}

The hybrid approach based edge detection can successfully reduce the poor speed issue with ant colony optimization. The color based gradients has the ability to remove the effect of the false edges while preserving the potential edges. In practice, the more the adaptive parameters are provided, the more conveniently the proposed method can be used. In this work, we employed two adaptive parameters which are mean square error and Pratt. The hybrid approach results are far much better than the existing results.

\section{REFERENCES}

[1] Tian, Jing, Weiyu Yu, and ShengliXie.2008. "An ant colony optimization algorithm for image edge detection." In IEEE Congress on Evolutionary Computation, pp.497-500. IEEE.

[2] Jing Tian, Weiyu Yu, and Shengli Xie.2008."An ant colony optimization algorithm for image edge detection"In IEEE World Congress on computational intelligence, pp.751- 756.IEEE.

[3] De Sian Lu, chien chang Chen.2008."Edge detection improvement by ant colony optimization"In science direct vol.29, no.4, pp.416-425.

[4] Bing Wang, Shao Sheng Fan.2009."An improved canny edge detection algorithm"In IEEE Second International Workshop on Computer Science and Engineering conference on 28-30oct. vol.1, pp.497-500.IEEE. 
[5] Wang Xiao, xue Hui.2010."An Improved Canny Edge Detection Algorithm Based on Predisposal Method for Image Corrupted by Gaussian Noise" In IEEE conference on 19-23 Sep, pp.113-116.IEEE.

[6] Yanhong Wang, Denghui Lian.2011. "Improved Image Edge Detection Algorithm Based on Fuzzy Sets" In Multimedia technology international conference on 2628 July2011, pp.2939-2941.

[7] Peng, Wu, and Chen Qichao.2012. "A novel SVM-based edge detection method." In Physics Procedia, pp.20752082 .

[8] Geng Xin, Chen Ke, Hu Xiaoguang.2012."An improved canny edge detection algorithm for Color image" In Informatics, no. 25-27, pp.113-117.IEEE.

[9] K. Rebai1, 2 and N. Achour, O. Azouaoui.2013."Hierarchical SVM Classifier for Road Intersection Detection and Recognition" In IEEE Conference on Open Systems (ICOS), pp100-105.IEEE.

[10] Bao, C. \& Sheng, C.2013."A parameterized logarithmic image processing method based on Laplacian of
Gaussian filtering for lung nodules enhancement in chest radiograph".In Instrumentation and Measurement, Sensor Network and Automation (IMSNA), 2nd International Symposium on. IEEE.

[11] Singh, Sanjay, Chandra Shekhar, and Anil Vohra.2013."Area optimized FPGA implementation of color edge detection." In International Conference Advanced Electronic Systems (ICAES), IEEE.

[12] Yong Chena, Min Xua, Huan-lin Liub, Wei-na Huanga, Jiang Xing.2014. "Improved image mosaic based on canny edge and an18-dimensional descriptor" In Optics communications, vol. 125, pp 4745-4750.

[13] Xiao hen Liu, Suping Fang.2015."A convenient and robust edge detection method based on ant colony optimization" In optics communications, vol.353, no.15, pp.147-157.

[14] Shahana N Youseph, Rajesh Roy Cherian.2015. "Pixel and edge based illuminant color estimation for image forgery detection "In international conference on information and communication technologies, vol.46, and pp.1635-1642. 